\title{
Erratum
}

\section{Modeling in Biopharmaceutics, Pharmacokinetics and Pharmacodynamics}

\author{
Panos Macheras and Athanassios Iliadis \\ C) Springer International Publishing Switzerland 2016 \\ P. Macheras, A. Iliadis, Modeling in Biopharmaceutics, Pharmacokinetics \\ and Pharmacodynamics, Interdisciplinary Applied Mathematics 30, \\ DOI 10.1007/978-3-319-27598-7
}

DOI 10.1007/978-3-319-27598-7_15

The original version of the book contained errors which have been corrected.

The corrections are given below:

\section{Chapter 9}

"Fractional Pharmacokinetics" is written by Dr. A. Dokoumetzidis, Faculty of Pharmacy, National and Kapodistrian, University of Athens.

The updated online version for this chapter can be found at

DOI: 10.1007/978-3-319-27598-7_9

\section{Chapter 10}

"Modeling and Simulation in Bioequivalence" is written by Dr. V. Karalis, Faculty of Pharmacy, National and Kapodistrian, University of Athens.

The updated online version for this chapter can be found at DOI: 10.1007/978-3-319-27598-7_10

The updated online version of the original book can be found at http://dx.doi.org/10.1007/978-3-319-27598-7 